\title{
Standardization of the technological parameters of vibratory abrasion as a tool for process reliability enhancement
}

\author{
Galina Prokopetz ${ }^{1}$, Anna Azarova ${ }^{1,{ }^{*}, \text { Anatoliy Prokopetz }}{ }^{1}$, and Elena Murugova ${ }^{1}$ \\ ${ }^{1}$ Don State Technical University, 1, Gagarin sq., 344003, Rostov-on-Don, Russia
}

\begin{abstract}
This paper deals with the general structure of the technological system of vibration abrasive processing and main issues of ensuring the stability of quality indicators formed in the final vibration abrasive processing. The research results of the working chamber oscillation amplitude influence and processing time on the wear rate of the processing environment are presented. The sample of determining the wear tolerance of the processing environment based on the roughness tolerance is presented.
\end{abstract}

\section{Introduction}

To maintain the specified quality level of manufactured parts during their production, it is necessary to ensure the stability of technological processes or operations, which, depending on the processing method, is provided by various means. For example, if a tool wearhas a large impact on the quality of manufactured parts during processing, then it is necessary to establish a pattern of changes in the quality index of the part formed during processing from this wear in order to take into account this destabilizing factor in the design and implementation of the technological processes. The impact of the tool wear can be controlled, for example, by adjusting the process system, replacing the tool when the critical wear value is reached, restoring the cutting ability of the tool, and so on. Moreover, it is needed to know the moment of intervention and correction in the process. This moment can be determined by any standard value (processing time, cutting length, number of processed parts, etc.), by active control of the process or the formed accuracy index, etc. However, in order to select a controlled parameter during active technological processes monitoring or to determine a normative value, it is necessary to have appropriate models that link the technological processes factors to its result.

It is particularly important to ensure the processing stability for finishing operations of the critical parts production, such as vibroabrasive processing [1-3] or vibrostrengthening treatment $[4,5]$, centrifugal-rotary processing $[6,7]$, both in the classical form, and combined methods using additional types of energy [2], among others applying coatings [8], etc.. These processing methods are used in various branches of machine-building

*Corresponding author:azarovaai@mail.ru 
production, for example, in the manufacture of transport machines [9], mining machines [10], in power engineering, etc.

As defined by one of the founders of vibration technology professor A. P. Babichev, physical essence of the process of vibratory treatment can be characterized as a complex influence of some factors caused by vibration and presence of relevant working environment, on the processed parts surface. The processing environment, the elements of which can have not only different sizes and shapes, but also different nature [11, 12] makes a complex movement, including its circulation and low-frequency vibrations, which in a whole provide the cutting process.

\section{Main part}

Vibration refers to the methods of surface dimensionless processing (except for the formation of the rounding edges radius, which is set as a dimensional allowance) and is mainly focused on the formation of quality surface indicators or the surface layer of the processed parts. The basic layout of the device for vibration processing is shown in Fig. 1.
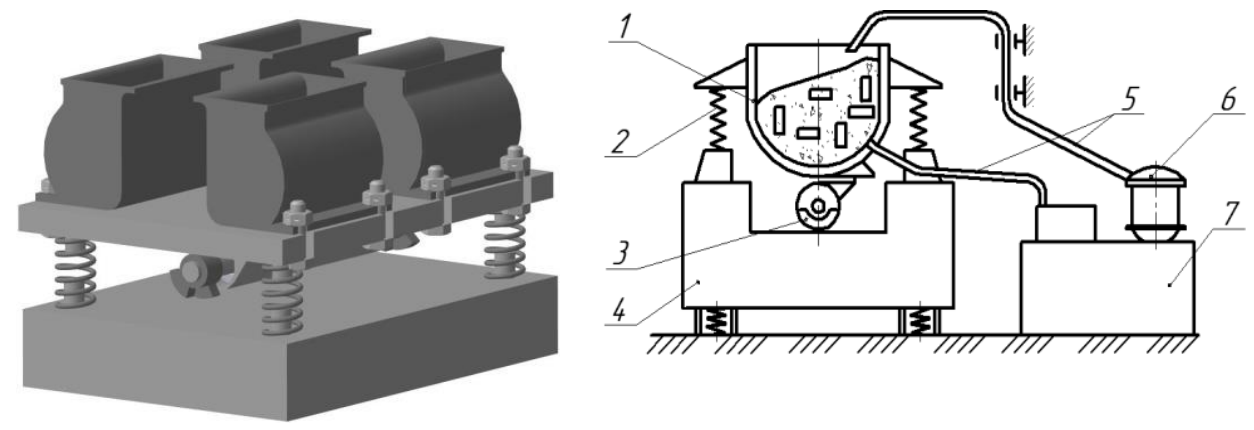

Fig. 1. The basic layout of the four-chambered installation and the device scheme for vibration processing: 1-the working chamber; 2-the elastic suspension; 3-the inertial vibrator; 4-the device body; 5-7 - the system of feeding and cleaning the process fluid.

During vibration treatment (Fig. 1) the processed parts are loaded into the working chamber 1 , filled with the working medium of the required characteristic, which is mounted on elastic elements 2, providing it with the ability to make fluctuations of the required characteristics.

The vibration abrasive treatment allows to clean castings or the parts surface from scale after heat treatment, remove burrs or flakes after stamping or parts machining, make the edges rounded, perform decorative and technological grinding, polishing, etc. Abrasion includes the system of fluid supply and cleaning.

This process was studied by prof. A.P. Babichev A. P. [3], prof. M.A. Tamarkin [1, 2, 7, 13], prof. V.A. Lebedev[12, 14, 15] and other scientists. However, a number of aspects still need to be examed.

A great advantage of vibration abrasive processing is the flexibility of the processing medium (in other words, the tool), which generally takes the form of the workpiece, which provides equal processing. However, at the same time, this fact complicates the analytical description of the process and requires a large amount of experimental research. Furthermore, studies of the vibration processes in order to normalize them are very relevant at this stage due to the high requirements to the technological processes reliability by vibration as well as the presence of numerous and diverse relationships between the elements of its technological system. 
At the first stage of the technological process analysis by vibration, it is advisable to structure the technological system. Analyzing the purpose and main tasks that are solved by the technological operation of vibratory abrasive processing, as well as the conditions for its implementation, you can imagine the structure of the technological system in the form shown in Fig. 2. All elements of the technological system can be divided into two groups:

- basic composition, without which the operation of vibroabrasive processing is completely impossible;

- elements that expand the technological capabilities of the technological system, including its reliability improvement.

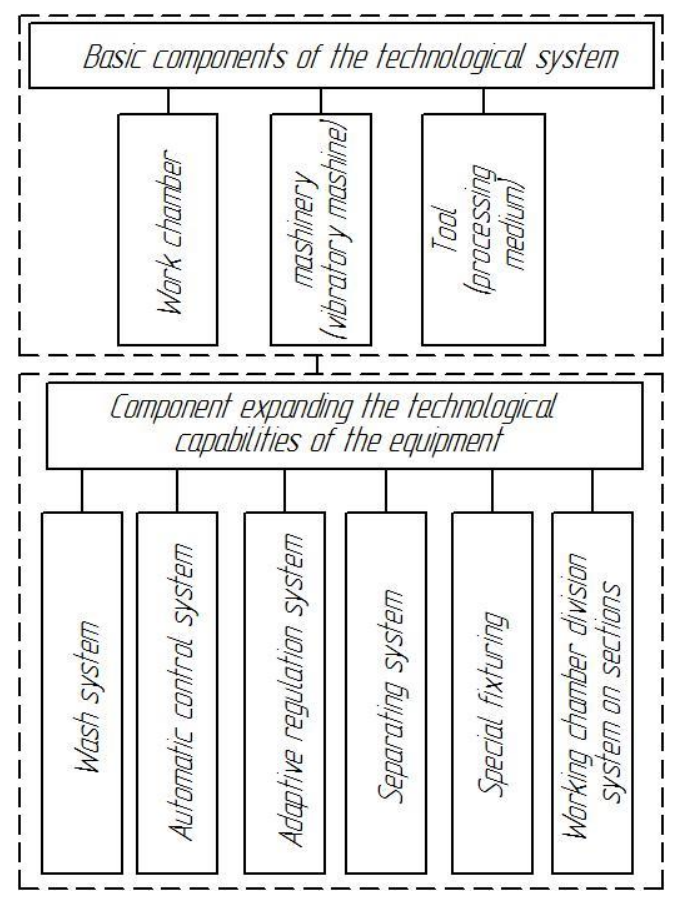

Fig. 2. Structure of the technological system (WC-working chamber).

The basic structure of the technological system of vibration processing includes (Fig. 2):

- equipment-vibrodevice (single-chamber, multi-chamber, torus, etc.);

- technological equipment in the form of working chambers of the corresponding form (or one working chamber);

- tool - processing medium with characteristics (type, composition, abrasive grit, granulation, etc.) corresponding to the characteristics of the processed parts (the most important are the material, pre-heat treatment, size and shape of the processed parts) and the purpose of processing.

The composition of the elements of the technological system that extends its technological capabilities depends on both the characteristics of the processed parts, and on organizational and economic restrictions (for example, the restriction on the performance of the technological operation). As for the processed parts, attention is payed to their material, the presence of pre-heat treatment, requirements for surface quality indicators, and their design features. In general, the technological system can include a number of different elements, as follows:

- washing system (see Fig. 1), which includes a pump, a system of fluid supply to the processing zone, a regulation system of flow process fluid rate; such system may also 
include a device for monitoring the degree of contamination of the process fluid, a cleaning (filtration) system, etc.;

- various means of automatic control of the process as a whole or its elements;

- adaptive process control system (the main goal of the system is to increase the reliability of the process operation, including minimization of the human factors on the processing result);

- systems of dividing the working chamber into sections, which are designed to divide a large and productive working chamber into sections, thereby creating separate smaller working chambers in which batches of different parts are processed; in this case, the processing is performed under the same conditions and under the same processing environment; however, the processing time may differ;

- fastening tooling:

- long or large enough machined parts that cannot make a circulation movement within the processing medium;

- parts where not all surfaces are processed;

- check test piece, etc.

- automatic separation system designed to separate the processed parts from the processing medium and return the latter to the processing zone, etc.

Each of the listed elements of the technological system has a greater or lesser impact on the reliability of the technological operation. However, as a rule, vibroabrasive processing is used in mass production, so when the degree of each of the elements influence on the reliability of the technological operation is taken into consideration, it is advisable to consider it for a period of time equal to processing time of the individual operation. In this case, it is possible to neglect a number of factors, for example, a wear coating, the inner cavity of the working chamber or items vibrodevice ware in general.

One of the elements that has minimal reliability, which is manifested within a single operation, is the working environment. The wear of the working environment leads to a change of volume loading, shape, and mass of particles in the manufacturing environment and, consequently, to the ratio changeof the volume of processing medium and the workpiece, the change in the energy characteristics of the manufacturing environment. The wear of the processing medium also depends on the processing mode: the amplitude and frequency of vibrations of the working chamber.

\section{Results}

The wear of the working environment is a function of the processing time. However, if the complexity of the technological operation is such that the wear of the processing medium within its duration does not exceed the allowable value, then this factor can also be considered insignificant. Otherwise, it must be taken into account, since the ratio between the volume of processed parts and the processing environment has a significant impact not only on the quality parameters of the part, but also on the performance of the process. For example, in Fig. 3 and 4 the dependence of the wear of the processing medium (abrasive e16TVwith an average granulation of $20 \mathrm{~mm}$ ) on the amplitude of vibrations and on the processing time. Experimental conditions: 4x10 UVG installation, working chamber volume-10 1, working chamber vibrational frequency $\mathrm{f}=33 \mathrm{~Hz}$. 


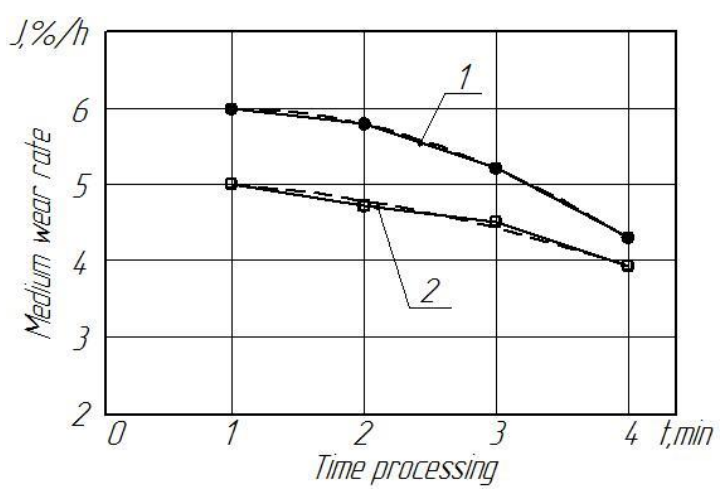

Fig. 3. Relationship between the wear rate of the processing medium and the processing time t: row $1-\mathrm{A}=2.5 \mathrm{~mm}$, regression: $\mathrm{J}=2,733 \mathrm{t} 3-16,8 \mathrm{t} 2+34.517 \mathrm{t}-18.7$ (R-squared value $\mathrm{R}=0.999$ ), row $2-\mathrm{A}=1.5$ $\mathrm{mm}, \mathrm{J}=2,667 \mathrm{t} 3-16,4 \mathrm{t} 2+33.933 \mathrm{t}-18$ (R-squared value $\mathrm{R}=0.999)$.

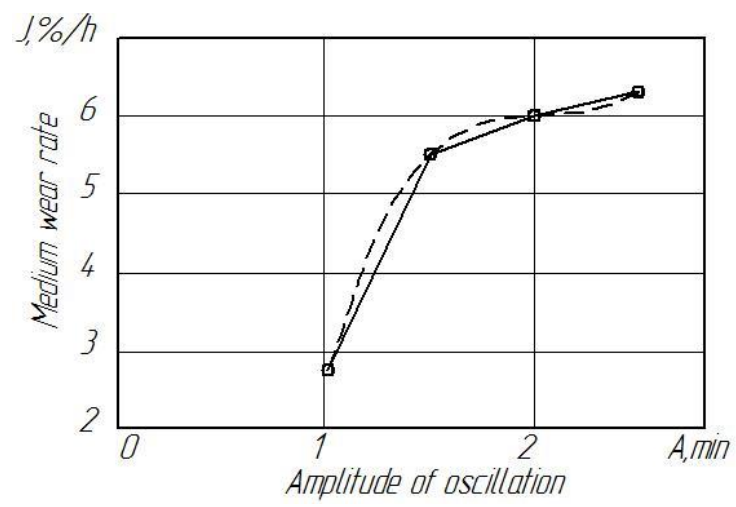

Fig. 4. Relationship of the wear rate of the processing medium to the oscillation amplitude of the working chamber A: time $\mathrm{t}=1$ hour, regression: $\mathrm{J}=-0,175 \mathrm{t} 2+0.305 \mathrm{t}+5.875$ (R-squared value $=0.999$ ).

From the graphical relationships shown in Fig. 3 and 4, it is seen that the relationship of the wear rate of the processing environment to the processing time and to the oscillation amplitude of the working chamber is determined. In symbolic form, second-and third degree polynomials describe these relationships. Then, by examining the relationship between the wear rate of the processed surface roughness and the loading volume of the working chamber, the allowance tolerance on the loading volume is fixed. In this case, the roughness of the processed parts will be within the tolerance. For example, to obtain a roughness of $\mathrm{Ra} \mathrm{3,} 2 \ldots$ 4,48 microns (in accordance with the Russian standard GOST 278973 ), the loading volume tolerance is 1,91 (from 5,4 to 7,21 ) or $18 \%$. Then according to the relationship shown in Fig. 2, the recovery of the loading volume (complete or partial replacement of the processing environment) with the amplitude of the working chamber $\mathrm{A}=2.5 \mathrm{~mm}$ is necessary to perform in 3.5 hours (Fig. 3).

When designing technological process, the range of acceptable values of process conditions and modes must be assigned in such a way as to ensure the specified quality of processing and stability within the scope of the technological operation. In this regard, it is necessary to divide these factors into significant and insignificant in terms of specific technological operations, the factors gradation influencing the surface quality of machined parts and machining performance, in terms of their impact, and the range of their permissible variation (tolerance) within the manufacturing operation. Thus, it is necessary to solve the problem of technological factors standardization in vibroabrasive processing. 


\section{References}

1. M. Tamarkin, E. Tishchenko, I. Viyalikov, Russian Engineering Research 35, 740-744 (2015)

2. A. Shishkina, V. Lebedev, M. Chaava, Bulletin of Tula State University Technical science 5, 278-282 (2016)

3. K. Hamouda, H. Bournine, M. Tamarkin, A. Babichev, D. Saidi, H. Amrou, Materials Science 52(2), 216-221 (2016) doi: 10.1007/s11003-016-9946-9

4. M. Tamarkin, E. Tishchenko, Russian Engineering Research 38(9), 726-727 (2018) doi: 10.3103/S1068798X18090277

5. Y. Kopilov, Journal of Machinery Manufacture and Reliability 39(3), 276-281 (2010)

6. A. Solovev, T. Van, M. Tamarkin, I. Panfilov, Physics and Mechanics of New Materials and Their Applications (PHENMA 2018), 334-336 (2018)

7. M. Tamarkin, E. Tishchenko, D. Kazakov, Proceedings of the International Conference on Mechanical, System and Control Engineering, 31-35 (2017) doi: 10.1109/ICMSC.2017.7959437

8. V. Ivanov, V. Lebedev, I. Panahin Journal of Friction and Wear 4, 339-342 (2014)

9. M. Tamarkin, E. Tishchenko, I. Chukarina, T. Chukarina, I. Chukarina, T. Sosnitzkaya Proceedings of the VIII International Scientific Siberian Transport Forum TransSiberia 1 (2019)

10. M. Popov, O. Hashash, D. Morgunov, La montagned'analyse et d'information bulletin (scientifique) 6, 107-112 (2017)

11. V. Butenko, V. Lebedev, I. Davidova, G. Serga, Proceedings of E3S Web of Conferences electronic edition, 01040 (2019)

12. A. Shishkina, V. Lebedev, E. Krupenia, Bulletin of the Rybinsk State Aviation Technological Academy named after P.A. Solovyov 2(41), 132-137 (2017)

13. M. Tamarkin, E. Tishchenko, A. Melnikov, E. Chernishov, Advances in Intelligent Systems and Computing 983, 661-669 (2019) doi: 10.1007/978-3-030-19868-8_64

14. A. Shishkina, V. Lebedev, M. Chaava, Bulletin of the Bryansk State Technical University 5(58), 42-49 (2017)

15. V. Lebedev, A. Sokolov, I. Davidova, Strengthening technologies and coatings 142(158), 54-58 (2018) 